\title{
VASCULAR CHANGES OVER TRABECULECTOMY BLEBS
}

\author{
PAUL T. K. CHEW, PETER G. WATSON and CAROLINE K. L. CHEE \\ Cambridge
}

\begin{abstract}
SUMMARY
Low-dose anterior segment fluorescein angiography was performed on 10 eyes before and after trabeculectomy with a limbal-based conjunctival flap. Post-trabeculectomy angiography shows that all successful trabeculectomies have an area of decreased capillary vascular perfusion over the bleb site not coincidental with the area of the bleb and that there is delayed filling of vessels running over the bleb.
\end{abstract}

The success of a trabeculectomy is dependent on the reduction of intraocular pressure which results from the drainage of the aqueous directly into the subconjunctival space. ${ }^{1,2}$ Since subconjunctival fibrosis is the commonest cause of trabeculectomy failure from proliferation of fibrovascular tissue scarring the bleb site,,${ }^{2-4}$ it is important to characterise the normal pattern of wound healing in a successful trabeculectomy. We report on 10 serial cases of trabeculectomy where low-dose anterior segment fluorescein angiography ${ }^{5}$ was performed on the eye pre-operatively and then at various intervals following the operation. The appearance of wound healing in these eyes was documented.

\section{PATIENTS AND METHODS}

Ten successive patients with primary open angle glaucoma who required trabeculectomy because of progressive visual field loss or inadequate control of intraocular pressure with beta blockers and pilocarpine were enrolled into the study. The study had been approved by the hospital ethics and research committees and informed consent was obtained from the patients.

All the patients had the operation performed by either P.G.W. or P.T.K.C. using a standardised technique. The selected trabeculectomy site was at 12 o'clock. A limbalbased conjunctival flap was fashioned, the episcleral tissue being included in the flap. A $4 \mathrm{~mm} \times 4 \mathrm{~mm}$ superficial flap was dissected just into the cornea. The deep scleral

From: Department of Ophthalmology, Addenbrooke's Hospital, Cambridge, UK.

Correspondence to: Paul T. K. Chew, Department of Ophthalmology, National University Hospital, 5 Lower Kent Ridge Road, Singapore 0511, Singapore. flap was dissected from behind forwards. The initial incision was behind the scleral spur, which was dissected from the iris root. The section which was removed therefore contained the whole trabecular meshwork. This specimen was sent for histological investigation. The superficial flap was replaced and sutured with two or three $10 / 0$ nylon sutures. The conjunction was hooded over the trabeculectomy site and sutured over the insertion of the superior rectus muscle. No antimetabolites were used for these cases.

All patients achieved intraocular pressures of less than $21 \mathrm{mmHg}$ without glaucoma medication throughout the follow-up period of 6 months.

Low-dose anterior segment fluorescein angiography (ASFA) was performed by the method described by Meyer and Watson ${ }^{5}$ using a Zeiss anterior segment camera modified for fluorescein angiography. Twenty per cent sodium fluorescein $(1.5 \mathrm{ml})$ was injected intravenously into an antecubital vein. A $720 \mathrm{~W}$ s high-cycle off-axis flash was used to take the angiograms at intervals of 1.5 seconds after injection of fluorescein. Kodak Tri X Pan $35 \mathrm{~mm}$ film was used.

ASFA was done 1 day before the operation during the first post-operative week, between the first and second post-operative month, and finally at the third to fourth month after surgery. The sequential angiograms were compared and the changes noted.

\section{RESULTS}

The pre-operative angiograms showed a normal limbal capillary arcade filling from deep scleral perforating arteries and normal radial episcleral arteries filling as described by Meyer. ${ }^{5,6}$ The order of filling was typically that the larger episcleral vessels filled from the anterior ciliary circulation and a slightly later filling of the limbal network came from scleral perforating arteries derived from the long posterior ciliary arterial supply (Fig. 1). There was minimal leakage of fluorescein into the extravascular space in the later stages of the angiogram (Fig. 2). The diffuse leak was homogeneous, of uniform distribution, and all the capillaries throughout the conjunctiva filled in the area under investigation. This was seen in all 10 eyes pre- 


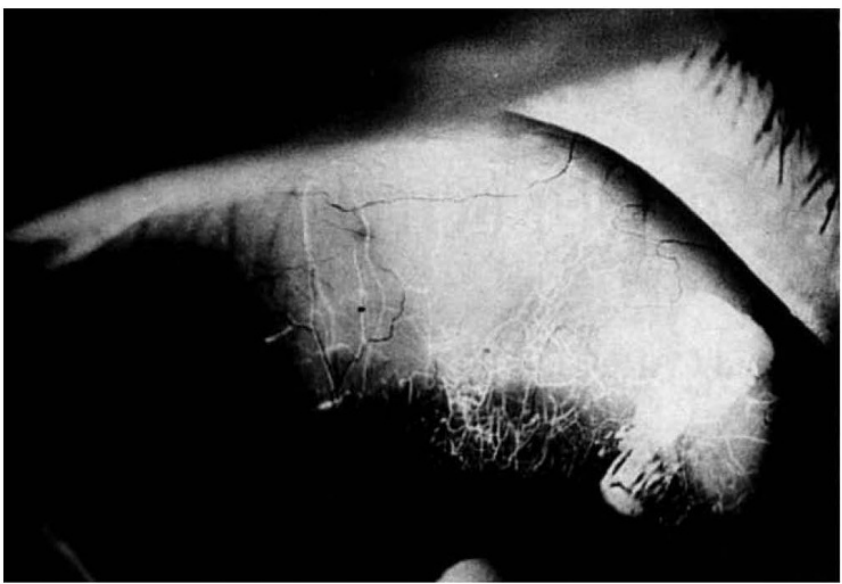

Fig. 1. Patient 1, pre-operative early phase angiogram. Normal anterior segment fluorescein angiogram. The normal limbal capillary arcades fill from conjunctival branches of the deep scleral perforating arteries. Posterior branches of the deep scleral perforators give rise to the radial episcleral arteries.

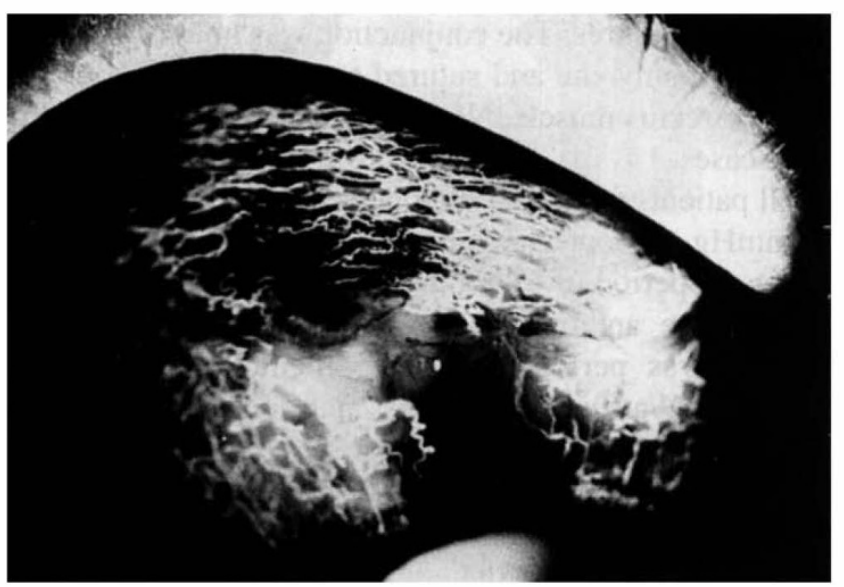

Fig. 3. Patient 1 , first post-operative week, early phase. An area of conjunctival and episcleral non-perfusion is seen over the trabeculectomy site.

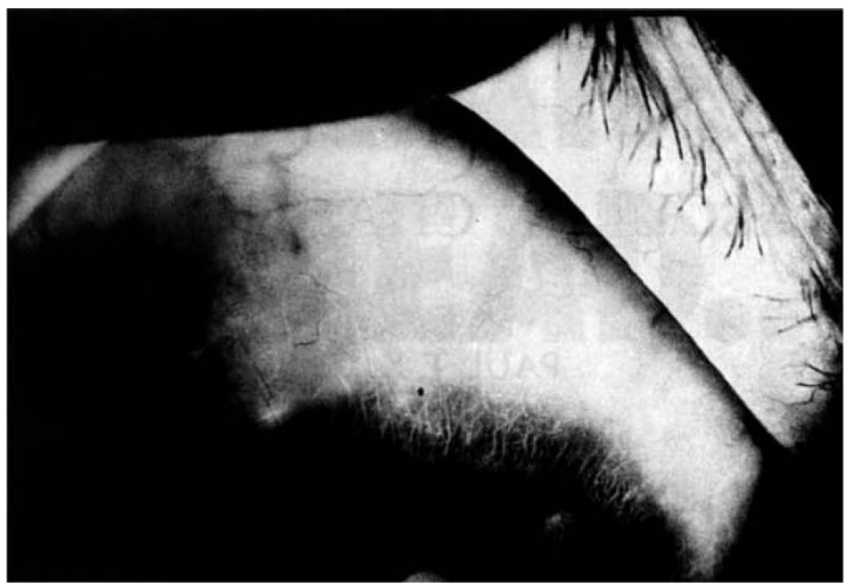

Fig. 2. Patient 1, pre-operative late phase. Fluorescein leakage from fenestral conjunctival vessels has occurred.

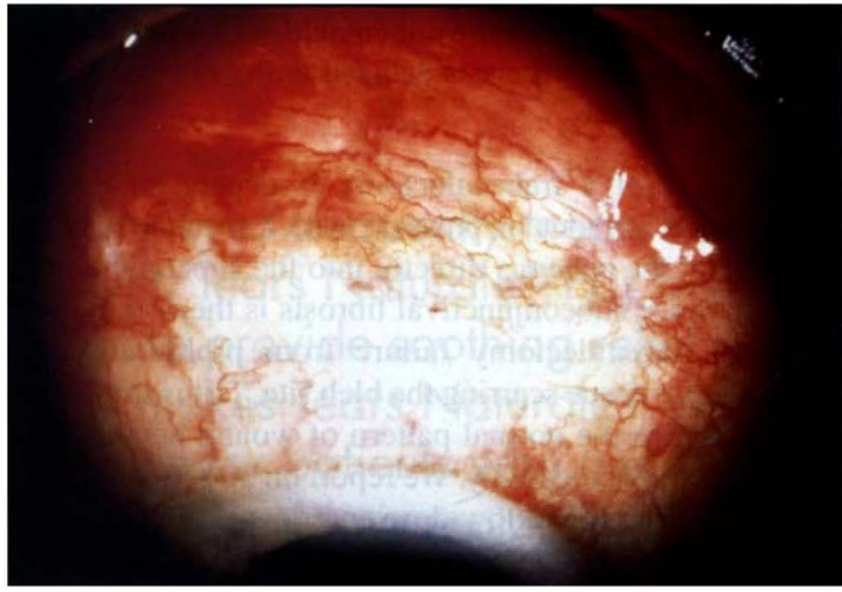

Fig. 4. Patient 1, first post-operative week. Colour photograph corresponding to Fig. 3. The area of non-perfusion in Fig. 3 is smaller than the bleb site seen in this photograph. The blood-filled limbal arcades seen in the colour photograph are not perfused on angiography.

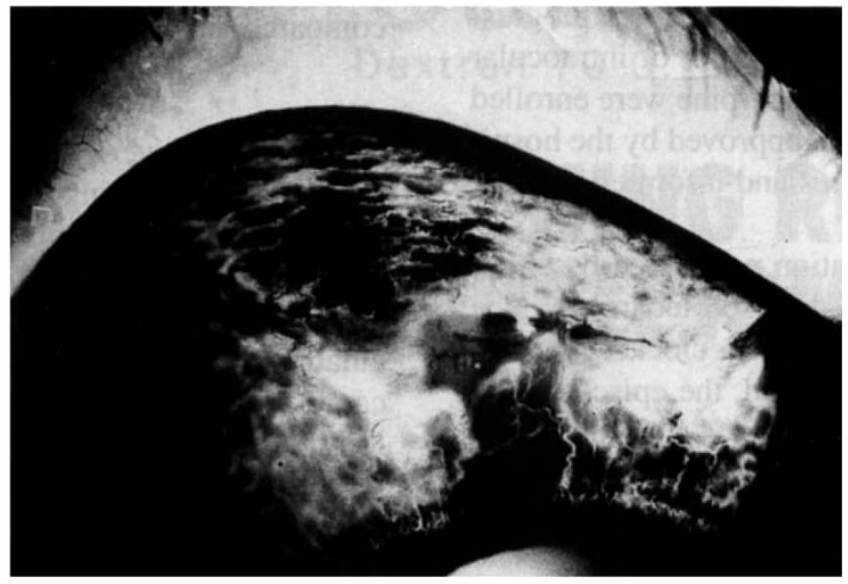

Fig. 5. Patient 1 , first post-operative week, late phase angiogram. A late-filling vessel appears over the non-perfused area. There is leakage of dye from surrounding vessels but the area of non-perfusion remains visible. 


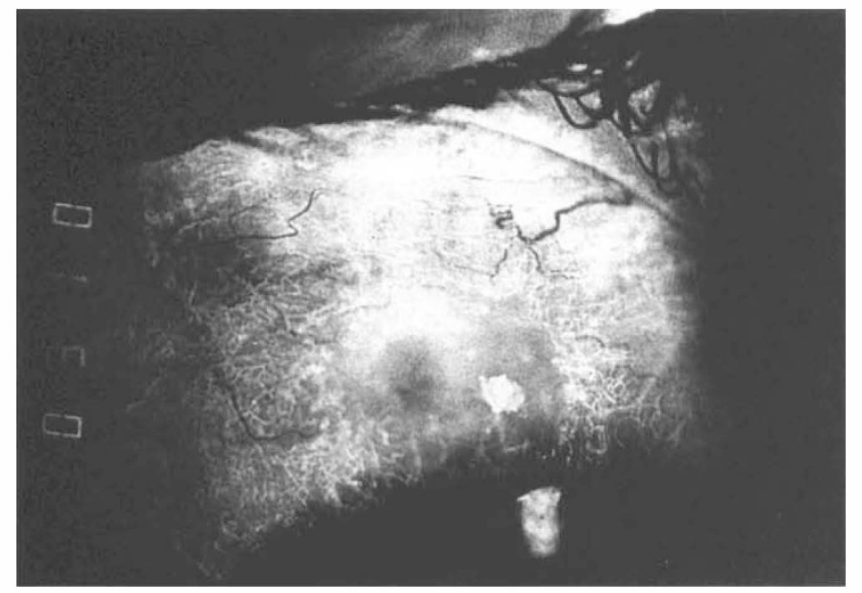

Fig. 6. Patient 2, first post-operative month, early phase angiogram. A leash of vessels surrounding a well-defined area of non-perfusion is seen.

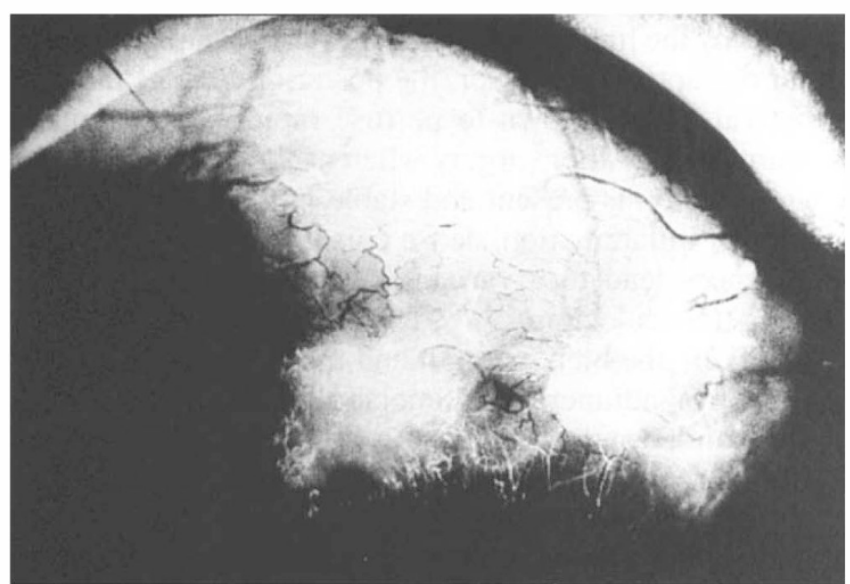

Fig. 8. Patient 1 , fourth post-operative month, late phase angiogram. There is an irregularly shaped patch of non-perfusion visible. There is leakage of dye into the area

operatively. Iris vessel fluorescence was also noted in some films. The first visible fluorescein was present about 10-15 seconds after injection and the last useful films were taken 20-40 seconds after first appearance.

\section{First Post-operative Week}

Angiography during the first post-operative week showed that there was early bleb formation visible in all cases on stereoscopic fluorescein angiogram viewing.

Non-perfused areas. There was a decrease in vascular perfusion at the bleb site with a dark patch typically showing the absence of fluorescein in both conjunctival and episcleral vessels in an irregular area over the operation site (Fig. 3). The area of non-perfused conjunctiva and episclera was often smaller than the actual bleb site. The borders of the non-perfused area and the bleb did not coincide (Figs. 3, 4). The non-perfused area was always contained inside the bleb. The edges of the non-perfused area were surrounded by leaking episcleral and conjunctival vessels which ran up to the edge of the bleb and formed loops at the edges with other vessels. Sometimes a large vessel ran over parts of the non-perfused portion of

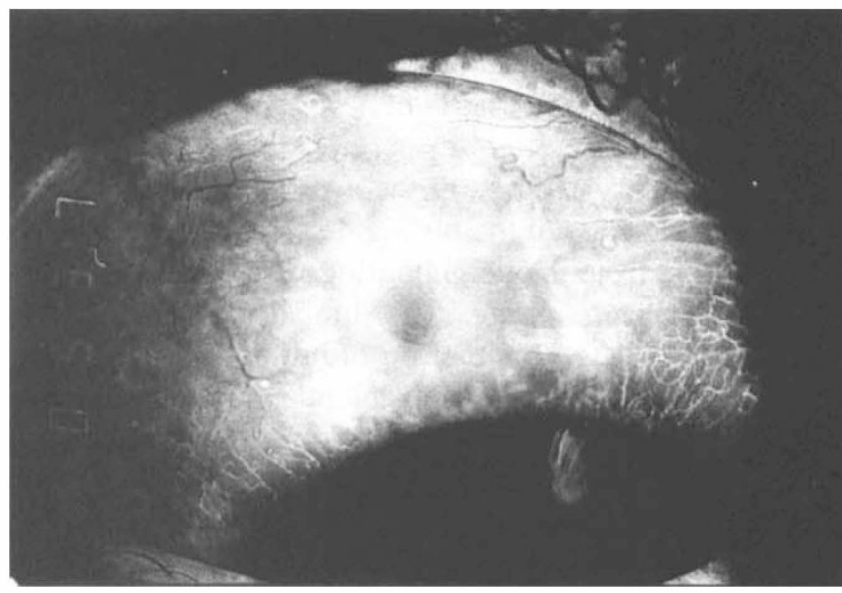

Fig. 7. Patient 2, first post-operative month, late phase angiogram. There is leakage of dye from neighbouring vessels. The non-perfused zone is still visible at 5 minutes after injection.

the bleb. This vessel, in spite of its larger size, showed slow flow and fluoresced late, showing up as a dark band running over a non-fluorescing area (Fig. 5).

Limbal arcade filling. There was poor perfusion of the limbal capillary arcades, with non-filling seen in the limbal arcades of 5 eyes. These remained non-perfused even in the late phase of the angiogram. In 6 eyes the arcade filled slowly and incompletely from the sides and, if a scleral perforating vessel was present at either side of the scleral flap, the limbal vessels gradually filled from the edge. In no case was a full limbal arcade circulation demonstrated. Deep scleral perforating vessels present pre-operatively directly over the surgical site where the scleral flap was raised were not seen after the operation (compare Fig. 1 and Fig. 3). Colour photography showed blood to be present in the limbal vessels of these eyes (Fig. 4) even though they were poorly perfused.

Episcleral arteries. There was late filling of the episcleral arteries noted in 4 of the eyes studied. These vessels were outside the non-perfused area. Filling occurred later than in the lateral or medial areas further removed from the surgical site. In the remaining eyes studied, episcleral vessels on the sides of the non-perfused area seemed to fill briskly and fluoresced well.

Iris perfusion. The sector of iris directly under the trabeculectomy site fluoresced later than the rest of the iris in 4 cases where iris pigment was light and good iris angiography was possible. The order of iris filling was delayed compared with the pre-operative films. The conjunctiva superior to the limbal-based flap incision was normally and fully perfused on angiography.

\section{First to Second Post-operative Month}

Angiograms after the first post-operative month showed similar features to those obtained in the first post-operative week, except that the perfusion pattern had been stabilised. There was an enlargement of the non-perfused areas and a leaking leash of new vessels around the bleb site in most of the cases (Figs. 6, 7).

Non-perfused areas. Conjunctival and episcleral 
vessels remained in an irregular distribution over the operation site. However, the non-fluorescing areas were larger and better defined. The leash of new vessels at the edge of the bleb was present in 5 eyes (including patient 2 illustrated in Fig. 6). The rest of the eyes had no new vessels present at the edge of the bleb and perfusion and leakage of dye came from the neighbouring perfused areas.

Limbal arcade filling. The limbal capillaries were poorly perfused, with either no filling or filling from the side in 8 eyes. Two eyes with scleral perforating arteries on either side of the trabeculectomy scleral flap did fill the limbus, but these did so late and poorly.

Episcleral arteries. The episcleral vessels under the bleb site were not perfused in any of the patients. There was evidence of reversal of flow direction in some of the perfused episcleral vessels alongside the non-perfused area in 2 cases.

Iris perfusion. This was similar to that seen in the first post-operative week.

\section{Third to Fourth Post-operative Month}

The appearance of the bleb was similar to that seen in the angiograms obtained during the first or second post-operative month (Fig. 8). The bleb edges became better defined but no further cases had developed a collar around the bleb site. Non-perfusion remained in an irregularshaped patch over the trabeculectomy site and limbal capillaries again filled from the side, but slowly and late compared with non-affected areas.

Dye leaked gradually into the bleb site from the surrounding periphery. This became more obvious in the later stages of the study, usually at 4-5 minutes after the injection of dye. By this time the episcleral vessels were already perfused with non-fluorescing blood. However, the area of non-perfusion still had less fluorescence than the surrounding areas.

\section{DISCUSSION}

In all the successful trabeculectomies studied here, the episcleral, conjunctival and limbal vessels failed to perfuse from the earliest time that it was possible to undertake a post-operative anterior segment fluorescein angiogram. The pattern was very similar in all the patients studied, in that the vessels in the region of the bleb, instead of gradually reperfusing, became less and less patent and eventually disappeared altogether to be replaced in most cases by a vascular cuff around the non-perfused segment. This is not the response which might be expected in recently traumatised tissue, in which new vessels enter the site together with the associated inflammatory fibroblasts to allow even healing of the wound. It would appear from the evidence of anterior segment fluorescein angiography that in the successful trabeculectomy the healing response has been curtailed for between a month and 6 weeks after the operation. Neovascular tufts are seen to be developing around the bleb site in the early films and those taken at 1 month. They leak and form loops with neighbouring vessels at the edge of the bleb site with occasional seafan-type appear- ances. These loops eventually form a stable collar or leash of vessels around the bleb site in some eyes, demarcating the area under which aqueous accumulates.

In those eyes where the inflammatory and wound healing response is less marked, then the collar does not form, giving rise to a diffuse bleb with poorly defined borders.

The non-perfused area seems to be due to the presence of normal aqueous under the conjunctiva. The formation of this non-perfused zone is not immediate but gradually increases over a period of 4-6 weeks, starting first with the smaller vessels but in some cases also involving the deep perforating vessels themselves.

Surgical trauma as a cause of lost perfusion seems to be partially responsible for the loss of perfusion to the limbal capillary arcades. The division of perforating vessels which normally supply the limbus when the scleral flap is raised would cause a loss of limbal vascular perfusion. However, there is a difference in the recovery of conjunctival and episcleral vessels compared with areas away from the operation site. The division of the conjunctival vessels by the limbal-based flap may cause temporary vascular disruption. However, the posterior edge of the conjunctival flap is shown to perfuse rapidly and normally within a month after surgery whereas the bleb-site-related non-perfusion is present and stable in the same eye.

Ocular inflammation, dense episcleral tissue and racial differences lead to a variable fibroblastic response following trabeculectomy. ${ }^{4.7-10}$ The greater this response, the more likely the bleb is to fail and the glaucoma to return. The use of adjunctive antimetabolites with trabeculectomy is aimed at suppressing this fibroblastic response. ${ }^{11.12}$ Anterior segment fluorescein angiography seems to be a method by which bleb site function can be assessed and the vascular component of the wound healing process followed. Having documentary evidence of the normal angiographic appearance of the bleb following uncomplicated successful trabeculectomy operations should make it possible to assess the value of antimetabolites in different situations and possibly predict the correct treatment or the need to augment treatment in difficult cases.

Key words: Anterior segment fluorescein angiography, Glaucoma, Trabeculectomy.

\section{REFERENCES}

1. Anderson DR. Glaucoma: the damage caused by pressure. Jackson Memorial Lecture. Am J Ophthalmol 1989;108: 485-95.

2. Watson PG, Jakeman C, Ozturk M, Barnett MF, Barnett F, Khaw KT. The complications of trabeculectomy: a 20 year follow up. Eye 1990;4:425-38.

3. Sturmer J, Broadway D, Hitchings RA. Young patient trabeculectomy: assessment of risk factors for failure. Ophthalmology 1993; 100:928-39.

4. Watson PG, Grierson I. The place of trabeculectomy in the treatment of glaucoma. Ophthalmology 1981;88:175-6.

5. Meyer P, Watson PG. Low dose fluorescein angiography of the conjunctiva and episclera. Br J Ophthalmol 1987;71: 2-10.

6. Meyer P. The circulation of the human limbus. Eye 1989;3: 121-7.

7. Lavin MJ, Wormald RPL, Migdal CS, Hitchings RA. The influence of prior therapy on the success of trabeculectomy. Arch Ophthalmol 1990;108:1543-8.

8. Broadway D, Grierson I, Hitchings RA. Adverse effects of 
topical antiglaucomatous medications on the conjunctiva. Br J Ophthalmol 1993;77:590-6.

9. Miller RD, Barber JC. Trabeculectomy in black patients. Ophthalmic Surg 1981;12:46-50.

10. Inaba $Z$. Long term results of trabeculectomy in the Japanese: an analysis by life-table method. Jpn J Ophthalmol 1982;26:361-73.
11. Miller MH, Rice NSC. Trabeculectomy combined with $\beta$ irradiation for congenital glaucoma. Br J Ophthalmol 1991; 75:584-90.

12. Smith MF, Sherwood MB, Koyle JW, Khaw PT. Results of intraoperative 5-fluorouracil supplementation on trabeculectomy for open-angle glaucoma. Am J Ophthalmol 1992; 114:737-41. 